\title{
Duality Theorems on an Infinite Network
}

\author{
マンハイム大学 Werner Oettli \\ 島根大学 山崎稀嗣 (Maretsugu Yamasaki)
}

\section{Introduction and Preliminaries}

Let $N=\{X, Y, K\}$ be an infinite network which is locally finite and has no self-loops. Here $X$ is the countable set of nodes, $Y$ is the countable set of arcs, $K: X \times Y \longmapsto\{-1,0,+1\}$ is the node-arc incidence matrix. Local finiteness means that $K(x, \cdot)$ has finite support in $Y$ for every $x \in X$.

We denote by $\mathcal{X}$ the set of all real-valued functions on $X$, and by $\mathcal{X}^{*}$ the set of all real-valued functions on $X$ with finite support. Likewise we denote by $\mathcal{Y}$ the set of all real-valued functions on $Y$, and by $\mathcal{Y}^{*}$ the set of all real-valued functions on $Y$ with finite support. For each $w \in \mathcal{Y}$, the divergence $\partial w \in \mathcal{X}$ is defined as

$$
\partial w(x):=\sum_{y \in Y} K(x, y) w(y) .
$$

For each $u \in \mathcal{X}$, the discrete derivative $d u \in \mathcal{Y}$ is defined as

$$
d u(y):=\sum_{x \in X} K(x, y) u(x)=u(b(y))-u(a(y)),
$$

where $a(y)$ is the initial node and $b(y)$ is the terminal node of arc $y$. Clearly, if $w \in \mathcal{Y}^{*}$, then $\partial w \in \mathcal{X}^{*}$, and if $u \in \mathcal{X}^{*}$, then $d u \in \mathcal{Y}^{*}$, since $N$ is locally finite.

For $w_{1}, w_{2} \in \mathcal{Y}$ with either $w_{1}$ or $w_{2}$ in $\mathcal{Y}^{*}$, we define the inner product

$$
<w_{1}, w_{2}>:=\sum_{y \in Y} w_{1}(y) w_{2}(y) .
$$

For $u, v \in \mathcal{X}$ with either $u$ or $v$ in $\mathcal{X}^{*}$, we define the inner product

$$
((u, v)):=\sum_{x \in X} u(x) v(x) .
$$

Note that the fundamental formula

$$
((u, \partial w))=<d u, w>
$$

holds if $u \in \mathcal{X}^{*}$ or $w \in \mathcal{Y}^{*}$. 
The space $\mathcal{X}$ can be identified with the product space $\mathbf{R}^{\boldsymbol{X}}$, and therefore can be given the product topology of $\mathbf{R}^{X}$. As usual, we call this the weak topology on $\mathcal{X}$. It is the topology of pointwise convergence, i.e., a sequence $\left\{\xi_{\nu}\right\}$ in $\mathcal{X}$ converges weakly to some $\xi \in \mathcal{X}$ if and only if $\xi_{\nu}(x) \rightarrow \xi(x)$ for all $x \in X$. If $\mathcal{X}$ is given the weak topology, then $\mathcal{X}^{*}$ becomes the topological dual of $\mathcal{X}$, which means that the continuous linear functionals on $\mathcal{X}$ are precisely those of the form $\langle u, \cdot\rangle$ with $u \in \mathcal{X}^{*}$. Henceforth, without exception, $\mathcal{X}$ will bear the weak topology. Likewise $\mathcal{Y}$ will always bear the weak topology, so that $\mathcal{Y}^{*}$ becomes the topological dual of $\mathcal{Y}$. We observe that the mappings $w \longmapsto \partial w$ and $u \longmapsto d u$ are continuous, if $\mathcal{X}$ and $\mathcal{Y}$ carry the weak topology. This follows from the fact that $K(x, \cdot)$ and $K(\cdot, y)$ have finite support.

\section{Weak Duality}

Let $F, G: \mathcal{Y} \longmapsto \mathbf{R} \cup\{+\infty\}$ be two convex, weakly lower semicontinuous functions which are mutually conjugate in the following sense:

For every $w_{1} \in \mathcal{Y}^{*}$,

$$
G\left(w_{1}\right)=\sup \left\{<w_{1}, w>-F(w) ; w \in \mathcal{Y}\right\}
$$

and for every $w_{2} \in \mathcal{Y}^{*}$,

$$
F\left(w_{2}\right)=\sup \left\{<w, w_{2}>-G(w) ; w \in \mathcal{Y}\right\} .
$$

From (2.1) and (2.2) it follows that

$$
<w_{1}, w_{2}>\leq G\left(w_{1}\right)+F\left(w_{2}\right)
$$

for all $w_{1}, w_{2}$ in $\mathcal{Y}$ with either $w_{1}$ or $w_{2}$ in $\mathcal{Y}^{*}$.

Now let $X_{1}$ and $X_{2}$ be two disjoint subsets $X$ such that $X=X_{1} \cup X_{2}$. Let $f_{1}, f_{2} \in \mathcal{X}$ be given such that the support of $f_{1}$ is contained in $X_{1}$ and the support of $f_{2}$ is contained in $X_{2}$. In order to introduce dual pairs of optimization problems on the network $N$ we define a primal objective function $E: \mathcal{Y} \longmapsto \mathbf{R} \cup\{+\infty\}$ as

$$
E(w):=F(w)-\left(\left(f_{1}, \partial w\right)\right) \text { for all } w \in \mathcal{Y},
$$

and we define a dual objective function $E^{*}: \mathcal{X} \longmapsto \mathbf{R} \cup\{+\infty\}$ as

$$
E^{*}(u):=-G(d u)+\left(\left(u, f_{2}\right)\right) \text { for all } u \in \mathcal{X} .
$$

In order to make $E$ well-defined we shall employ the following hypothesis:

$$
f_{1} \in \mathcal{X}^{*} \text {. }
$$


In order to make $E^{*}$ well-defined we shall employ the following hypothesis:

$$
f_{2} \in \mathcal{X}^{*} \text {. }
$$

However, if $E$ is restricted to $\mathcal{Y}^{*}$, then (E.1) is not needed, and if $E^{*}$ is restricted to $\mathcal{X}^{*}$, then (E.2) is not needed. The functions $E$ and $-E^{*}$ are convex and weakly lower semicontinuous, with values in $\mathbf{R} \cup\{+\infty\}$.

If $w \in \mathcal{Y}$ is a flow on the arcs $y \in Y$, then $F(w)$ may be considered as a generalized energy of $w$. And if $u \in \mathcal{X}$ is a potential on the nodes $x \in X$, then $G(d u)$ may be considered as a generalized Dirichlet sum of $u$.

We consider two pairs of optimization problems as follows:

To the primal problem

$$
\inf \left\{E(w) ; w \in \mathcal{Y}, \partial w(x)=f_{2}(x) \text { on } X_{2}\right\}
$$

we associate the dual problem

$$
\sup \left\{E^{*}(u) ; u \in \mathcal{X}^{*}, u(x)=f_{1}(x) \text { on } X_{1}\right\} .
$$

And to the primal problem

$$
\inf \left\{E(w) ; w \in \mathcal{Y}^{*}, \partial w(x)=f_{2}(x) \text { on } X_{2}\right\}
$$

we associate the dual problem

$$
\sup \left\{E^{*}(u) ; u \in \mathcal{X}, u(x)=f_{1}(x) \text { on } X_{1}\right\} .
$$

We adopt the convention that the infimum over the empty set equals $+\infty$, and the supremum over the empty set equals $-\infty$. Obviously the only difference between $(P)$ and $\left(P_{0}\right)$ and between $(D)$ and $\left(D_{0}\right)$ consists in the underlying spaces. In case $N$ is a finite network, a similar problem was treated in [1], p. 162.

Henceforth we denote by $V(P), V\left(D_{0}\right), V\left(P_{0}\right), V(D)$ the optimal values of the problems $(P),\left(D_{0}\right),\left(P_{0}\right),(D)$ respectively. We shall study duality relations between $(P)$ and $\left(D_{0}\right)$ and between $\left(P_{0}\right)$ and $(D)$, and describe an application of our results to the potential theory on locally finite networks.

We have the following weak duality result:

Theorem 2.1 (1) Assume that (E.1) holds. Then $V(P) \geq V\left(D_{0}\right)$.

(2) Assume that (E.2) holds. Then $V\left(P_{0}\right) \geq V(D)$.

Proof. (1) The claim is obviously true, if $(P)$ or $\left(D_{0}\right)$ have no feasible solutions. So let $w$ and $u$ be feasible solutions for $(P)$ and $\left(D_{0}\right)$ respectively. Then

$$
\begin{aligned}
E(w)-E^{*}(u) & =F(w)+G(d u)-\left(\left(f_{1}, \partial w\right)\right)-\left(\left(u, f_{2}\right)\right) \\
& =F(w)+G(d u)-((u, \partial w)) \\
& =F(w)+G(d u)-<d u, w> \\
& \geq 0
\end{aligned}
$$


from (2.3), since $u \in \mathcal{X}^{*}$. Thus $E(w) \geq E^{*}(u)$ for all feasible $w$ and $u$, which implies $V(P) \geq V\left(D_{0}\right)$. The proof of $(2)$ is similar.

From (E.1) it follows that problem $\left(D_{0}\right)$ has a feasible solution, i.e., there exists $u \in \mathcal{X}^{*}$ such that $u=f_{1}$ on $X_{1}$. Likewise we have

Proposition 2. 1 Assume that (E.2) holds and that $X_{1} \neq \emptyset$. Then problem $\left(P_{0}\right)$ has a feasible solution, i.e., there exists $w \in \mathcal{Y}^{*}$ such that $\partial w(x)=f_{2}(x)$ on $X_{2}$.

Proof. Fix $x_{0} \in X_{1}$. For every $a \in X_{2}$ select a finite path $p_{a} \in \mathcal{Y}^{*}$ from $x_{0}$ to $a$, i.e., $p_{a}$ is the path index of a path from $x_{0}$ to $a$ (cf. [6]). Then $p_{a}$ is a unit flow from $x_{0}$ to $a$, i.e., $\partial p_{a}(a)=+1, \partial p_{a}\left(x_{0}\right)=-1$ and $\partial p_{a}(x)=0$ for all other $x$. Let us consider

$$
w(y):=\sum_{a \in X_{2}} f_{2}(a) p_{a}(y)
$$

Then $w(y)$ is well-defined, since $f_{2}$ has finite support in $X_{2}$, and it is easily seen that $w$ has the requested properties.

For later use we denote by $\varepsilon_{A}$ the characteristic function of a subset $A \subset X$, i.e., $\varepsilon_{A}(x)=1$ for $x \in A$ and $\varepsilon_{A}(x)=0$ for $x \in X \backslash A$.

\section{A General Duality Theorem}

Our main tool will be a general duality result studied in [5](cf. [4]). We prepare it below for the sake of completeness.

Let $\mathcal{U}$ be a real vector space, let $\mathcal{Z}$ be a locally convex topological vector space, and let $\mathcal{W}$ be the topological dual of $\mathcal{Z}$. Let $\varphi: \mathcal{U} \rightarrow \mathbf{R} \cup\{+\infty\}$ and $\psi: \mathcal{Z} \rightarrow \mathbf{R} \cup\{-\infty\}$ be given. Let $C$ be a nonempty subset of $\mathcal{U}$ and $Q$ be a nonempty subset of $\mathcal{Z}$. Let $T$ be a transformation from $\mathcal{U}$ into $\mathcal{Z}$.

Let us consider the following general extremum problem (V) and its dual problem $\left(\mathrm{V}^{*}\right)$ :

$$
\begin{gathered}
V:=\inf \{\varphi(\xi)-\psi(T \xi) ; \xi \in C, T \xi \in Q\} \\
V^{*}:=\sup \left\{\psi^{*}(\zeta)-\varphi_{T}^{*}(\zeta) ; \zeta \in \mathcal{W}\right\}
\end{gathered}
$$

where

$$
\begin{aligned}
\psi^{*}(\zeta) & :=\inf \{\zeta(\eta)-\psi(\eta) ; \eta \in Q\} \\
\varphi_{T}^{*}(\zeta) & :=\sup \{\zeta(T \xi)-\varphi(\xi) ; \xi \in C\}
\end{aligned}
$$

It is always true that $V \geq V^{*}$. We have by [5] 
Theorem 3. 1 Assume that the set

$$
\mathcal{E}:=\{(z, s) \in \mathcal{Z} \times \mathbf{R} ; z=\eta-T \xi, s \geq \varphi(\xi)-\psi(\eta), \xi \in C, \eta \in Q\}
$$

is convex and closed in $\mathcal{Z} \times \mathbf{R}$. If $V$ is finite, then $V=V^{*}$ holds and there exists $\xi \in C$ such that $T \xi \in Q$ and $V=\varphi(\xi)-\psi(T \xi)$.

Proof. Clearly, $V=\inf \{s ;(0, s) \in \mathcal{E}\}$. Let $V$ be finite. Then $(0, V) \in \mathcal{E}$, since $\mathcal{E}$ is closed, and this gives the existence of $\xi \in C$ with the claimed property. In order to prove $V \leq V^{*}$, let $t<V$. Then $(0, t) \notin \mathcal{E}$. Hence from the strong separation theorem there exists $(\zeta, \tau) \in \mathcal{W} \times \mathbf{R}$ such that

$$
\zeta(0)+\tau t<\zeta(z)+\tau s \quad \forall(z, s) \in \mathcal{E} .
$$

Since $(0, V+r) \in \mathcal{E}$ for all $r \geq 0$, we obtain from (3.1) that $\tau>0$. Dividing (3.1) by $\tau$ and rewriting $\zeta / \tau$ as $\zeta$, we obtain

$$
t \leq \zeta(z)+s \quad \forall(z, s) \in \mathcal{E}
$$

hence in particular

$$
t \leq \zeta(\eta-T \xi)+\varphi(\xi)-\psi(\eta)
$$

for all $\xi \in C, \eta \in Q$, and therefore $t \leq \psi^{*}(\zeta)-\varphi_{T}^{*}(\eta) \leq V^{*}$. Since $t<V$ was arbitrary, we obtain $V \leq V^{*}$.

\section{Duality between $(P)$ and $\left(D_{0}\right)$}

We are going to derive the strong duality relation $V(P)=V\left(D_{0}\right)$ from Theorem 3.1. We assume (E.1) and specify the data of Theorem 3.1 as follows:

$\mathcal{U}:=\mathcal{Y}, \mathcal{Z}:=\mathcal{X}, \mathcal{W}:=\mathcal{X}^{*} ; C:=\mathcal{Y}, Q:=\left\{\eta \in \mathcal{X} ; \eta=f_{2}\right.$ on $\left.X_{2}\right\}$

$$
T \xi:=\partial \xi, \quad \varphi(\xi):=F(\xi), \quad \psi(\eta):=\left(\left(f_{1}, \eta\right)\right), \quad \zeta(\eta):=((\eta, \zeta))
$$

for all $\xi \in \mathcal{Y}, \eta \in \mathcal{X}, \zeta \in \mathcal{X}^{*}$. Then we have for all $\xi \in \mathcal{Y}$

$$
\varphi(\xi)-\psi(T \xi)=F(\xi)-\left(\left(f_{1}, \partial \xi\right)\right)=E(\xi)
$$

Therefore $V=V(P)$. For all $\zeta \in \mathcal{X}^{*}$ we have

$$
\begin{aligned}
\varphi_{T}^{*}(\zeta) & =\sup \{((\zeta, \partial \xi))-F(\xi) ; \xi \in C\} \\
& =\sup \{<d \zeta, \xi>-F(\xi) ; \xi \in \mathcal{Y}\}=G(d \zeta) \\
\psi^{*}(\zeta) & =\inf \left\{\left(\left(\zeta-f_{1}, \eta\right)\right) ; \eta \in Q\right\} \\
& =\inf \left\{\left(\left(\zeta-f_{1}, \eta \varepsilon_{X_{2}}+\eta \varepsilon_{X_{1}}\right)\right) ; \eta \in Q\right\} \\
& =\left(\left(\zeta, f_{2}\right)\right)+\inf \left\{\left(\left(\zeta-f_{1}, \eta \varepsilon_{X_{1}}\right)\right) ; \eta \in Q\right\}
\end{aligned}
$$


Therefore $\psi^{*}(\zeta)=\left(\left(\zeta, f_{2}\right)\right)$ if $\zeta-f_{1}=0$ on $X_{1}$, and $\psi^{*}(\zeta)=-\infty$ otherwise. Thus $V^{*}=V\left(D_{0}\right)$.

In order to apply Theorem 3.1 we need another hypothesis:

$$
\text { The level sets }\{\xi \in \mathcal{Y} ; F(\xi)-<w, \xi>\leq \alpha\} \quad(\alpha \in \mathbf{R})
$$

are weakly compact in $\mathcal{Y}$ for all $w \in \mathcal{Y}^{*}$.

Theorem 4. 1 Assume that (E.1) holds, that $V(P)$ is finite and that (H.1) is satisfied. Then $V(P)=V\left(D_{0}\right)$ and problem $(P)$ has an optimal solution.

Proof. The result follows from Theorem 3.1. We only have to show that the convex set

$$
\mathcal{E}=\{(z, s) \in \mathcal{X} \times \mathbf{R} ; z=\eta-\partial \xi, s \geq \varphi(\xi)-\psi(\eta), \xi \in C, \eta \in Q\}
$$

is closed in $\mathcal{X} \times \mathbf{R}$, where $\mathcal{X}$ bears the weak topology. Since the set $X$ of nodes is countable, $\mathcal{X}$ is a metrizable space under the weak topology (cf. [2], p. 32). Therefore the weak closedness in $\mathcal{X}$ means the sequential weak closedness (cf. [2], p. 20). Thus we have to show that $\mathcal{E}$ is sequentially closed. Let $\left\{\left(z_{n}, s_{n}\right)\right\}$ be a sequence in $\mathcal{E}$ such that $z_{\boldsymbol{n}} \rightarrow \bar{z}$ pointwise and $s_{n} \rightarrow \bar{s}$ in $\mathbf{R}$. There exist $\xi_{n} \in C$ and $\eta_{n} \in Q$ such that $z_{n}=\eta_{n}-\partial \xi_{n}$, $s_{n} \geq F\left(\xi_{n}\right)-\left(\left(f_{1}, \eta_{n}\right)\right)$. Then

$$
\begin{aligned}
s_{\boldsymbol{n}} & \geq F\left(\xi_{\boldsymbol{n}}\right)-\left(\left(f_{1}, \partial \xi_{\boldsymbol{n}}+z_{\boldsymbol{n}}\right)\right) \\
& =F\left(\xi_{\boldsymbol{n}}\right)-<d f_{1}, \xi_{\boldsymbol{n}}>-\left(\left(f_{1}, z_{\boldsymbol{n}}\right)\right) .
\end{aligned}
$$

Because of (E.1), $\left\{\left(\left(f_{1}, z_{n}\right)\right)\right\}$ converges to $\left(\left(f_{1}, \bar{z}\right)\right)$. Thus the sequence $\left\{\left(\left(f_{1}, z_{n}\right)\right)\right\}$ remains bounded. Since $\left\{s_{n}\right\}$ is also bounded, we see that the sequence $\left\{F\left(\xi_{n}\right)-<d f_{1}, \xi_{n}>\right\}$ is bounded from above. Thus, because of (H.1), all $\xi_{n}$ are contained in a weakly compact subset of $\mathcal{Y}$. Since the set $Y$ of arcs is countable, $\mathcal{Y}$ is metrizable under the weak topology. Hence the weak compactness of a closed set in $\mathcal{Y}$ means the sequential weak compactness (cf. [2], p. 21). So, by choosing a subsequence if necessary, we may assume that $\left\{\xi_{n}\right\}$ converges pointwise to some $\bar{\xi} \in C$. Then $\partial \xi_{n} \rightarrow \partial \bar{\xi}$ pointwise, and $\eta_{n}=\partial \xi_{n}+z_{n} \rightarrow \bar{\eta}=\partial \bar{\xi}+\bar{z} \in Q$ pointwise. Thus $\bar{z}=\bar{\eta}-\partial \bar{\xi}$ and $\bar{s} \geq F(\bar{\xi})-\left(\left(f_{1}, \bar{\eta}\right)\right)$, since $F$ is weakly lower semicontinuous. Thus $(\bar{z}, \bar{s}) \in \mathcal{E}$, and $\mathcal{E}$ is closed.

\section{$5 \quad$ Duality between $\left(P_{0}\right)$ and $(D)$}

Now we are going to derive the duality relation $V\left(P_{0}\right)=V(D)$. We assume (E.2) and specify the data of Theorem 3.1 as follows:

$\mathcal{U}:=\mathcal{X}, \mathcal{Z}:=\mathcal{Y}, \mathcal{W}:=\mathcal{Y}^{*} ; C:=\left\{\xi \in \mathcal{X} ; \xi=f_{1}\right.$ on $\left.X_{1}\right\}, Q:=\mathcal{Y} ;$

$$
T \xi:=d \xi, \psi(\eta):=-G(\eta), \varphi(\xi):=-\left(\left(\xi, f_{2}\right)\right), \zeta(\eta):=-<\eta, \zeta>
$$


for all $\xi \in \mathcal{X}, \eta \in \mathcal{Y}, \zeta \in \mathcal{Y}^{*}$.

Then for all $\xi \in \mathcal{X}$ there holds

$$
\varphi(\xi)-\psi(T \xi)=-\left(\left(\xi, f_{2}\right)\right)+G(d \xi)=-E^{*}(\xi) .
$$

Therefore $V=-V(D)$. For all $\zeta \in \mathcal{Y}^{*}$ there holds

$$
\begin{aligned}
\psi^{*}(\zeta) & =\inf \{-<\eta, \zeta>+G(\eta) ; \eta \in Q\}=-F(\zeta) \\
\varphi_{T}^{*}(\zeta) & =\sup \left\{-<d \xi, \zeta>+\left(\left(\xi, f_{2}\right)\right) ; \xi \in C\right\} \\
& =\sup \left\{\left(\left(\xi,-\partial \zeta+f_{2}\right)\right) ; \xi \in C\right\} \\
& =\sup \left\{\left(\left(\xi \varepsilon_{X_{2}},-\partial \zeta+f_{2}\right)\right) ; \xi \in C\right\}-\left(\left(f_{1}, \partial \zeta\right)\right)
\end{aligned}
$$

Therefore $\varphi_{T}^{*}(\zeta)=-\left(\left(f_{1}, \partial \zeta\right)\right)$ if $-\partial \zeta+f_{2}=0$ on $X_{2}$, and $\varphi_{T}^{*}(\zeta)=+\infty$ otherwise. Hence $\psi^{*}(\zeta)-\varphi_{T}^{*}(\zeta)=-E(\zeta)$ for all $\zeta \in \mathcal{Y}^{*}$ which are feasible for $\left(P_{0}\right)$, and $\psi^{*}(\zeta)-\varphi_{T}^{*}(\zeta)=-\infty$ otherwise. Thus $V^{*}=-V\left(P_{0}\right)$.

We prepare

Proposition 5.1 Let $\left\{\xi_{n}\right\} \subset \mathcal{X}$, and let $a \in X$. If $\left\{d \xi_{n}\right\}$ converges pointwise and if $\left\{\xi_{n}(a)\right\}$ converges, then $\left\{\xi_{n}\right\}$ converges pointwise to some $\xi \in \mathcal{X}$.

Proof. For every $x \in X$ select a finite path $p_{x} \in \mathcal{Y}^{*}$ from $a$ to $x$. Then

$$
<d \xi_{n}, p_{x}>=\left(\left(\xi_{n}, \partial p_{x}\right)\right)=\xi_{n}(x)-\xi_{n}(a) .
$$

Since $\left\{<d \xi_{n}, p_{x}>\right\}$ converges and $\left\{\xi_{n}(a)\right\}$ converges, $\left\{\xi_{n}(x)\right\}$ converges, too. Since this holds for every $x \in X,\left\{\xi_{n}\right\}$ converges pointwise to some $\xi \in \mathcal{X}$.

We further introduce the following hypothesis:

$$
\text { The level sets }\{\eta \in \mathcal{Y} ; G(\eta)-<\eta, w>\leq \alpha\} \quad(\alpha \in \mathbf{R})
$$

are weakly compact in $\mathcal{Y}$ for all $w \in \mathcal{Y}^{*}$.

Theorem 5.1 Assume that (E.2) holds, that $V(D)$ is finite, that $X_{1} \neq \emptyset$, and that (H.2) is satisfied. Then $V\left(P_{0}\right)=V(D)$ and problem $(D)$ has an optimal solution.

Proof. This follows from Theorem 3.1. As in the proof of Theorem 4.1, we shall show that the convex set

$$
\mathcal{E}=\{(z, s) \in \mathcal{Y} \times \mathbf{R} ; z=\eta-d \xi, s \geq \varphi(\xi)-\psi(\eta), \xi \in C, \eta \in Q\}
$$

is sequencially weakly closed in $\mathcal{Y} \times \mathbf{R}$. Let $\left\{\left(z_{n}, s_{n}\right)\right\}$ be a sequence in $\mathcal{E}$ such that $z_{n} \rightarrow \bar{z}$ pointwise, and $s_{n} \rightarrow \bar{s}$. There exist $\xi_{n} \in C$ and $\eta_{n} \in Q$ such that

$$
z_{n}=\eta_{n}-d \xi_{n}, s_{n} \geq-\left(\left(\xi_{n}, f_{2}\right)\right)+G\left(\eta_{n}\right) .
$$


By Proposition 2.1, there exists $w \in \mathcal{Y}^{*}$ such that $\partial w=f_{2}$ on $X_{2}$. From $\xi_{n} \in C$ we obtain then

$$
\begin{aligned}
\left(\left(\xi_{n}, f_{2}\right)\right) & =\left(\left(\xi_{n}, \partial w\right)\right)-\left(\left(f_{1}, \partial w\right)\right) \\
& =<d \xi_{n}, w>-\left(\left(f_{1}, \partial w\right)\right) \\
& =<\eta_{n}-z_{n}, w>-\left(\left(f_{1}, \partial w\right)\right)
\end{aligned}
$$

Thus

$$
s_{\boldsymbol{n}} \geq<z_{\boldsymbol{n}}, w>+\left(\left(f_{1}, \partial w\right)\right)-<\eta_{\boldsymbol{n}}, w>+G\left(\eta_{\boldsymbol{n}}\right) .
$$

Since $\left\{\left\langle z_{n}, w>\right\}\right.$ converges to $\left\langle\bar{z}, w>\right.$, we see that the sequence $\left.\left\{-<\eta_{\boldsymbol{n}}, \boldsymbol{w}\right\rangle+G\left(\eta_{\boldsymbol{n}}\right)\right\}$ is bounded from above. Using hypothesis (H.2), by the same reasoing as in the proof of Theorem 4.1, we may assume that $\left\{\eta_{n}\right\}$ converges pointwise to some $\bar{\eta} \in \mathcal{Y}$. Then $\left\{d \xi_{n}\right\}$ converges also pointwise to $\bar{\eta}-\bar{z}$. Since $X_{1} \neq \emptyset$ and $\xi_{n} \in C$, we see that $\xi_{n}(a)=f_{1}(a)$ for some $a \in X_{1}$. From Proposition 5.1 it follows that $\left\{\xi_{n}\right\}$ converges poitwise to some $\bar{\xi} \in C$. Then $\left\{d \xi_{n}\right\}$ converges pointwise to $d \bar{\xi}$, so that $d \bar{\xi}=\bar{\eta}-\bar{z}$. Altogether we obtain that

$$
\bar{z}=\bar{\eta}-d \bar{\xi}, \bar{s} \geq-\left(\left(\bar{\xi}, f_{2}\right)\right)+G(\bar{\eta})
$$

since $G$ is weakly lower semicontinuous. Thus $(\bar{z}, \bar{s}) \in \mathcal{E}$, and $\mathcal{E}$ is closed.

\section{Applications}

As applications of our duality results, we obtain generalizations of some fundamental inverse relations from [3] and [6] which play important roles in the discrete potential theory (cf. [7]).

We let $F$ and $G$ be as before. In addition we assume that $F$ and $G$ are nonnegative and symmetric, and that $G$ is homogeneous of degree $q>1$ and $G$ is homogeneous of degree $p>1$, with $1 / p+1 / q=1$.

In connection with problems $\left(P_{0}\right)$ and $(D)$ we choose $f_{2}=0$ (so that (E.2) holds), and we assume that $f_{1} \neq 0$ (so that $X_{1} \neq \emptyset$ ). For all $\eta \in \mathcal{Y}^{*}$ we let $I(\eta):=\left(\left(f_{1}, \partial \eta\right)\right)$. We define

$$
\begin{aligned}
\beta & :=\inf \left\{p G(d u) ; u \in \mathcal{X}, u=f_{1} \text { on } X_{1}\right\} \\
\alpha_{0} & :=\inf \left\{q E(\eta) ; \eta \in \mathcal{Y}^{*}, \partial \eta=0 \text { on } X_{2}, I(\eta)=1\right\}
\end{aligned}
$$

It is obvious that $\beta \geq 0, \alpha_{0} \geq 0$, and

$$
V(D)=\frac{-1}{p} \beta .
$$


Moreover we have

$$
\begin{aligned}
V\left(P_{0}\right) & =\inf \left\{F(w)-I(w) ; w \in \mathcal{Y}^{*}, \partial w=0 \text { on } X_{2}\right\} \\
& =\inf \left\{\inf \left\{|t|^{q} F(\eta)-t ; \eta \in \mathcal{Y}^{*}, \partial \eta=0 \text { on } X_{2}, I(\eta)=1\right\} ; t \in \mathbf{R}\right\} \\
& =\inf \left\{\frac{|t|^{q} \alpha_{0}}{q}-t ; t \in \mathbf{R}\right\} \\
& =-\frac{1}{p} \alpha_{0}^{-p / q}
\end{aligned}
$$

So, if $V(D)$ is finite and $\neq 0$, the duality relation $V\left(P_{0}\right)=V(D)$ takes the form

$$
\beta^{1 / p} \alpha_{0}^{1 / q}=1
$$

From Theorem 5.1 we obtain therefore

Corollary 6.1 Assume that $\beta$ is finite and $\neq 0$, and that (H.2) is satisfied. Then $\beta^{1 / p} \alpha_{0}^{1 / q}=1$.

On the other hand, if we define

$$
\begin{aligned}
\beta_{0} & :=\inf \left\{p G(d u) ; u \in \mathcal{X}^{*}, u=f_{1} \text { on } X_{1}\right\} \\
\alpha & :=\inf \left\{q E(\eta) ; \eta \in \mathcal{Y}, \partial \eta=0 \text { on } X_{2}, I(\eta)=1\right\}
\end{aligned}
$$

then $V\left(D_{0}\right)=-\beta_{0} / p$ and $V(P)=-\alpha^{-p / q} / p$. We obtain from Theorem 4.1

Corollary 6. 2 Assume that (E.1) and (H.1) are satisfied, and that $\alpha$ is finite and $\neq 0$. Then $\beta_{0}^{1 / p} \alpha^{1 / q}=1$.

From Corollary 6.1 we can obtain Theorem 5.1 in [3]. To be more specific, assume that $A$ is an arbitrary subset of $X$, and $B$ is a nonempty subset of $X$ which is disjoint with $A$. Let us take

$$
X_{1}:=A \cup B, X_{2}:=X \backslash(A \cup B), f_{1}:=\varepsilon_{B}, f_{2}=0 .
$$

Then

$$
I(\eta)=\sum_{x \in B} \partial \eta(x)
$$

In case $\partial \eta=0$ on $X_{2}, I(\eta)$ is called the strength of $\eta$ on $B$. Let, as in [3],

$$
\begin{aligned}
d_{p}(A, B) & :=\inf \{p G(d u) ; u \in \mathcal{X}, u=0 \text { on } A, u=1 \text { on } B\}=\beta \\
d_{q, 0}^{*}(A, B) & :=\inf \left\{q F(\eta) ; \eta \in \mathcal{Y}^{*}, \partial \eta=0 \text { on } X \backslash(A \cup B), I(\eta)=1\right\}=\alpha_{0}
\end{aligned}
$$

Notice that Corollary 6.1 gives a sufficient condition for the validity of the inverse relation

$$
\left(d_{p}(A, B)\right)^{1 / p} \cdot\left(d_{q, 0}^{*}(A, B)\right)^{1 / q}=1 .
$$


Observe that from $\eta \in \mathcal{Y}^{*}$ and $\partial \eta=0$ on $X \backslash(A \cup B)$ it follows that

$$
\sum_{x \in B} \partial \eta(x)=-\sum_{x \in A} \partial \eta(x)
$$

Remark 6.1 Let $r \in \mathcal{Y}$ be strictly positive and take $F$ as

$$
F(w):=\frac{1}{q} \sum_{y \in Y} r(y)|w(y)|^{q} .
$$

Then we have

$$
G(w)=\frac{1}{p} \sum_{y \in Y} r(y)^{1-p}|w(y)|^{p} .
$$

Notice that $p G(d u)=D_{p}(u)$ (Dirichlet sum of $u$ of order $p$ ) and $q F(w)=H_{q}(w)$ (the energy of $w$ of order $q$ ) (cf. [3]). We see that $F$ satisfies (H.1) and that $G$ satisfies (H.2).

\section{References}

[1] E. Blum and W. Oettli, Mathematische Optimierung, Springer-Verlag, 1975.

[2] N. Dunford and J. T. Schwartz, Linear Operators Part I: General Theory, John Wiley and Sons, 1957.

[3] T. Nakamura and M. Yamasaki, Generalized extremal length of an infinite network, Hiroshima Math. J. 6(1976), 95 -111.

[4] R. T. Rockafellar, Conjugate Duality and Optimization (Regional Conference Series in Applied Mathematics, Vol. 16), SIAM, Philadelphia, 1974.

[5] M. Yamasaki, Some generalizations of duality theorems in mathematical programming problems, Math. J. Okayama 14(1969), $69-81$.

[6] M. Yamasaki, Extremum problems on an infinite network, Hiroshima Math. J. $\mathbf{5}(1975), 223-250$

[7] M. Yamasaki, Parabolic and hyperbolic infinite networks, Hiroshima Math. J. 7(1976), $135-146$. 